\title{
Psychosocial factors in adults with chronic kidney disease: characteristics of pilot participants in the Tasmanian Chronic Kidney Disease study
}

Charlotte M McKercher ${ }^{1 *}$, Alison J Venn', Leigh Blizzard ${ }^{1}$, Mark R Nelson ${ }^{1}$, Andrew J Palmer ${ }^{1}$, Michael A Ashby ${ }^{1,2}$, Jennifer $\mathrm{L} \mathrm{Scott}^{1,3}$ and Matthew D Jose $\mathrm{e}^{1,2,4}$

\begin{abstract}
Background: Psychosocial factors including depression, anxiety and lower social support are common in patients with chronic kidney disease (CKD). However the influence of these potentially modifiable risk factors on morbidity and mortality in this renal population is unknown. The Tasmanian Chronic Kidney Disease study is a prospective cohort study which aims to examine the influence of both biomedical and psychosocial factors on disease progression, decision making and length and quality of life in adults with severe CKD, prior to kidney replacement therapy (KRT). This paper describes the recruitment, baseline characteristics and initial follow-up of pilot participants.

Methods: Adults aged > 18 years with stage 4 CKD (eGFR $15-29 \mathrm{mls} / \mathrm{min} / 1.73 \mathrm{~m}^{2}$ ) and not receiving dialysis were recruited via treating physicians. Measures included depression (9-item Patient Health Questionnaire), anxiety (Beck Anxiety Inventory) and social support (Multidimensional Scale of Perceived Social Support). Primary outcomes were kidney disease progression, use of KRT and health-related quality of life (Kidney Disease and Quality of Life Short Form and the EQ-5D).

Results: Of those invited $(n=105), 49$ provided consent and completed baseline assessment. There were no significant differences between responders and non-responders in age, gender and socio-economic status (all p > 0.05). Participants were predominantly male (63.3\%) with a mean age of $72.6 \pm 10.2$ years. Mean serum creatinine was $241 \pm 62 \mu \mathrm{mol} / \mathrm{L}$ with mean eGFR $22 \pm 5 \mathrm{mls} / \mathrm{min} / 1.73 \mathrm{~m}^{2}$. Prevalence of major depression and moderate to severe anxiety was $10 \%$ and $9 \%$ respectively. Less severe depression and fewer anxiety symptoms were associated with higher health-related quality of life. Follow-up at 10-months showed CKD progression in 34\% of participants (use of KRT in 16\%, stage 5 CKD without KRT in 18\%), one death, with the remainder stable at CKD stage 3 or 4.

Conclusions: Results indicate that a larger prospective study is feasible and has the capacity to examine the influence of biomedical and psychosocial factors on kidney disease progression, use of dialysis and transplantation, and salient personal and economic outcomes. Findings have the potential to provide an evidence base for revising healthcare provision in order to optimize the care of patients with CKD.
\end{abstract}

Keywords: Anxiety, Chronic kidney disease, Cohort, Depression, Health-related quality of life, Social support

\footnotetext{
* Correspondence: Charlotte.McKercher@utas.edu.au

${ }^{1}$ Menzies Research Institute Tasmania, University of Tasmania, Hobart,

Tasmania, Australia

Full list of author information is available at the end of the article
} 


\section{Background}

Chronic kidney disease (CKD) is a progressive disease associated with decreased quality of life, progression to end-stage kidney disease (ESKD), and increased risk of cardiovascular disease and mortality. Evidence from various chronic disease settings indicates that psychosocial factors including depression, anxiety and social isolation have a significant impact on quality of life, biological factors and disease progression [1-3]. Targeted psychosocial interventions have been shown to improve quality of life, treatment compliance, physical functioning and reduce the risk of cardiovascular disease $[1,4]$. However, despite the increasing social and economic burden of CKD [5], our understanding of the influence of psychosocial factors on patients in this population is limited. Examining the influence of potentially modifiable psychosocial factors on the progression of disease and management of those with advanced CKD has the capacity to identify areas for psychosocial intervention in this vulnerable and growing population.

Research examining psychosocial factors in renal populations is relatively new and has focussed predominantly on patients with ESKD. Depression is associated with increased mortality and decreased quality of life in patients on dialysis [6-8], however few studies have examined the influence of depression on disease progression and adverse outcomes in patients with CKD prior to the initiation of kidney replacement therapy (KRT) (dialysis or transplantation) $[9,10]$. These studies indicate that clinical depression [9] and elevated depressive symptoms [10] are independent predictors of clinical outcomes, including initiation of dialysis, hospitalization or death in CKD patients. In contrast, Kop and colleagues found that higher depressive symptoms were associated with subsequent acute kidney injury but not renal function decline, hospitalization or initiation of dialysis [11].

In comparison to depression, there is a paucity of research examining the influence of anxiety and social isolation in patients with kidney disease. Anxiety disorders are often perceived to represent symptoms of depression rather than independent conditions and therefore have been relatively understudied in this medical population. There is a high prevalence of anxiety in patients treated with hemodialysis [12] however the impact of anxiety in CKD patients not on dialysis is unknown. Finally, while lower perceived social support is associated with decreased quality of life $[13,14]$ and increased mortality in ESKD [13-15] this relationship has not been investigated in CKD prior to KRT.

The aim of the current pilot study is to examine the feasibility and efficacy of the Tasmanian Chronic Kidney Disease study, a prospective cohort study of non-dialyzed adults with severe CKD living in Tasmania, Australia [16] with a minimum follow-up of 3-years. The main objectives of this study are to examine the relative influence of both biomedical and psychosocial factors on kidney disease progression, use of KRT and overall length and quality of life. Findings will have direct translation into clinical practice (by identifying psychosocial factors as important predictors of outcomes), healthcare service provision (by focusing health services on quality of life rather than extending life at all costs) and healthcare policy. The current paper describes the study design, the key clinical and psychosocial characteristics of pilot participants at baseline, and clinical progression at 10-month follow-up.

\section{Methods}

\section{Inclusion criteria and recruitment}

All adults living in Tasmania aged $>18$ years with an estimated glomerular filtration rate (eGFR) $15-29 \mathrm{mls} / \mathrm{min} /$ $1.73 \mathrm{~m}^{2}$ on a single reading in the previous 3-months and not receiving dialysis between 1 July 2010 and 9 August 2011 were eligible for inclusion in the pilot study. Potential participants were identified by use of the Clinical Audit Tool [17] or through a direct request to pathology services to identify any patient with an eGFR 15-29 mls/ $\mathrm{min} / 1.73 \mathrm{~m}^{2}$ for the first time during the recruitment period. Recruitment from primary care was irrespective of (and separate to) referral to a renal physician. Eligible participants were sent an enrolment package in the mail by their treating doctor. This consisted of an information booklet and a letter requesting return of a signed consent form indicating participation. If consent forms were not returned within two weeks potential participants were followed-up by phone. The pilot study was approved by the Tasmanian Health and Medical Human Research Ethics Committee (protocol H0011543).

\section{Procedure}

Participants were sent self-report questionnaires prior to attending a study clinic where baseline clinical and laboratory data including height, weight, blood pressure and hemoglobin were measured and functional capacity was assessed. Participants unable to attend a clinic had measurements taken at their place of residence. Clinic assessments took approximately 1.5 hours.

\section{Measures}

\section{Psychosocial and sociodemographic factors}

Psychosocial factors were self-reported using the Patient Health Questionnaire [18], the Beck Anxiety Inventory [19] and the Multidimensional Scale of Perceived Social Support [20]. All measures have previously been validated in people with kidney disease and were used to facilitate comparisons within the literature. The Beck Anxiety Inventory was included in order to distinguish symptoms of anxiety from those of depression. 
Patient Health Questionnaire (PHQ-9) The PHQ-9 is a 9-item screening and diagnostic tool devised to assess the presence and severity of depression. Respondents rate how often they have experienced depressive thoughts or feelings over the prior 2-weeks on a scale ranging from 0 (not at all) to 3 (nearly every day) with total scores ranging from 0 to 27. Scores $\geq 10$ have sensitivity and specificity of $92 \%$ for a diagnosis of major depression in patients on hemodialysis [21].

Beck Anxiety Inventory (BAI) The BAI is a 21-item screening tool designed specifically to distinguish symptoms of anxiety from those of depression. It also includes an item examining fear of death (thanatophobia) which may influence decision making concerning treatment. Respondents rate the extent to which each symptom has bothered them in the past month, ranging from 0 (not at all) to 3 (severely, I could barely stand it). Total scores range from 0 to 63 with scores of $\geq 16$ indicating moderate to severe levels of anxiety [22]. The BAI has demonstrated high internal consistency and adequate discriminant validity in older patients in primary care [23].

\section{Multidimensional Scale of Perceived Social Support} (MSPSS) The MSPSS is a 12-item inventory which assesses overall perceived social support. The scale contains three subscales, family, friends, and significant others, which are averaged to comprise an overall score. Scores are reported on seven-point scale, with higher scores indicating higher perceived social support. Lower levels of perceived social support derived using this scale have predicted poorer survival in hemodialysis patients [24].

Sociodemographic and health information including marital status, education level, employment status, household income and smoking history was collected via questionnaire. Area-level socio-economic status (SES) based on residential postcode was derived using the Australian Bureau of Statistics socioeconomic index for areas (SEIFA) [25]. The SEIFA is a summary of four indices designed to measure different aspects of SES based on questions asked in the 2001 Census of Population and Housing [25]. The Index of Disadvantage is derived from attributes including low income, low educational attainment, high unemployment and employment in unskilled occupations. For the current analysis postcodes were divided into four quartiles according to ranking in this index, the first quartile representing greater disadvantage compared with other geographical areas.

\section{Biomedical factors}

Information regarding the number and severity of medical comorbidities was obtained from the participant's treating doctor using a modified Charlson comorbidity index (CMI) [26]. Body mass index (BMI) $\left(\mathrm{kg} / \mathrm{m}^{2}\right)$ was derived from objectively measured height and weight and categorized according to standard BMI cut-points [27]. Cognitive function was assessed objectively using the Modified Mini-Mental State Examination [28] and via self-report using the cognitive function subscale of the Kidney Disease Quality of Life short-form (KDQOLSF 1.3) [29]. Functional status was reported by the participant's treating doctor using the Karnofsky Performance Scale [30] while functional ability was selfreported using the Activities of Daily Living index [31] and the Instrumental Activities of Daily Living scale [32]. Functional capacity was assessed using hand grip dynometry (average of two measurements) and gait speed (time taken to walk 5-metres) using standardized protocols. Healthcare decision making was self-reported using the Autonomy Preference Index [33] and the Krantz Health Opinion Survey [34].

Modified Mini-Mental State Examination (3MS) The $3 \mathrm{MS}$ is an interviewer administered test of global cognitive function which assesses orientation, attention, language, and memory. Scores range from 0 to 100 with higher scores indicating better cognitive function. The $3 \mathrm{MS}$ is more sensitive to early cognitive changes than the mini-mental state with scores $<80$ having a sensitivity of $97 \%$ and a specificity of $91 \%$ for detecting dementia [28].

Kidney Disease Quality of Life short-form (KDQOLSF 1.3) The KDQOL-SF combines the 36 generic items of the SF-36 with 43 kidney disease-targeted items. Eleven subscales are defined from the kidney disease-targeted items, resulting in a total of 19 subscales (i.e., eight generic and 11 targeted to patients with kidney disease and treated by dialysis). Responses are weighted and transformed to scores ranging from 0 to 100 , with higher scores indicating better self-assessed health-related quality of life. The SF-36 has two subscales, the physical component summary (SF-36 PCS) and the mental component summary (SF-36 MCS). The KDQOL-SF 1.3 cognitive function subscale has been validated in people with CKD with a median score of 87 [35].

Karnofsky Performance Scale (KPS) The KPS is a physician-rated measure of a patient's functional status. Scores range from 0 to 100 with a score of 100 indicating full capacity to carry out normal activities. Scores < 70 indicate that some level of assistance is needed to carry out daily activities. Lower KPS scores have predicted higher risk of mortality in patients with ESKD [36].

Activities of Daily Living (ADL) The ADL assesses functional status as a measure of the patient's ability to perform activities of daily living independently. Scores 
range from 0 to 6 with a score of 6 indicating full function. The scale is sensitive to changes in declining health status and has been used effectively among older adults in a variety of care settings.

Instrumental Activities of Daily Living (IADL) The IADL assesses independent living skills in order to identify current functioning as well as improvement or deterioration over time. Patients are scored according to their highest level of functioning across 8 domains with a summary score ranging from 0 (low function, dependent) to 8 (high function, independent).

Autonomy Preference Index (API) The API identifies dimensions of autonomy by measuring patients' preferences for making medical decisions (decision-making subscale) and their desire to be informed (informationseeking subscale). Higher scores indicate stronger preferences in favor of decision making or information-seeking.

Krantz Health Opinion Survey (KHOS) The KHOS measures preferences regarding information about healthcare (information-seeking subscale) and active involvement in healthcare (behavioral-involvement subscale). While the API measures preference for healthcare informationseeking behavior for decisions not necessarily already made, the KHOS examines manifest information-seeking and behavioral involvement. Higher scores indicate that the patient is proactive in seeking healthcare information and professional medical assistance.

\section{Outcome measures}

Primary outcome measures were kidney disease progression, use of KRT and subjective health-related quality of life. Kidney disease progression was measured continuously according to decrease in mean eGFR and categorically per the Kidney Disease Outcomes Quality Initiative staging system [37] using the Modification of Diet in Renal Disease formula [38]. Use of KRT was obtained by consented linkage to the Australia and New Zealand Dialysis and Transplant Registry (ANZDATA). Health-related quality of life was assessed using both a psychometric health status measure (KDQOL-SF 1.3) and a utility-based measure (EQ-5D) [39]. Health status measures discriminate levels of functioning between groups and detect changes in function over time while utility-based measures are utilized in cost-effectiveness analyses and to guide decisions regarding resource allocation. The EuroQol Group's EQ-5D is commonly used to calculate quality adjusted life years (QALYs) [40] and has been used extensively to assess utility-based quality of life in CKD patients [41]. The EQ5D has five dimensions (mobility, self-care, usual activities, pain/discomfort, and anxiety/depression). In the current analysis responses to the EQ-5D 3-level version were dichotomized as 'no problem' versus 'some/severe problems'.

\section{Statistical analysis}

Data are presented descriptively as mean (standard deviation) or median (range) for continuous variables, and frequency (percentage) for categorical variables. Betweengroup differences were compared with Student $t$ test or the chi-square test, where appropriate. Associations between psychosocial and clinical variables with healthrelated quality of life were calculated using Spearman's correlation coefficient. Analyses were conducted using Stata/IC version 12.0 (Statacorp, 2011) with statistical comparison treated as significant at $\alpha=0.05$ (two-tailed).

\section{Results}

An overview of recruitment and retention is presented in Figure 1. Of the 105 eligible patients approached to participate, $18 \%(n=19)$ declined participation, $21 \%(n=22)$ did not respond and $13 \%(n=14)$ were lost to follow-up. Subsequently $48 \%(n=50)$ of those approached provided informed consent and 47\% ( $\mathrm{n}=49)$ completed baseline assessments. There were no significant differences between responders and non-responders in mean age, gender distribution and Index of Disadvantage quartiles (all $\mathrm{p}>0.05$ ).

The baseline sociodemographic, clinical and laboratory characteristics of pilot participants are displayed in

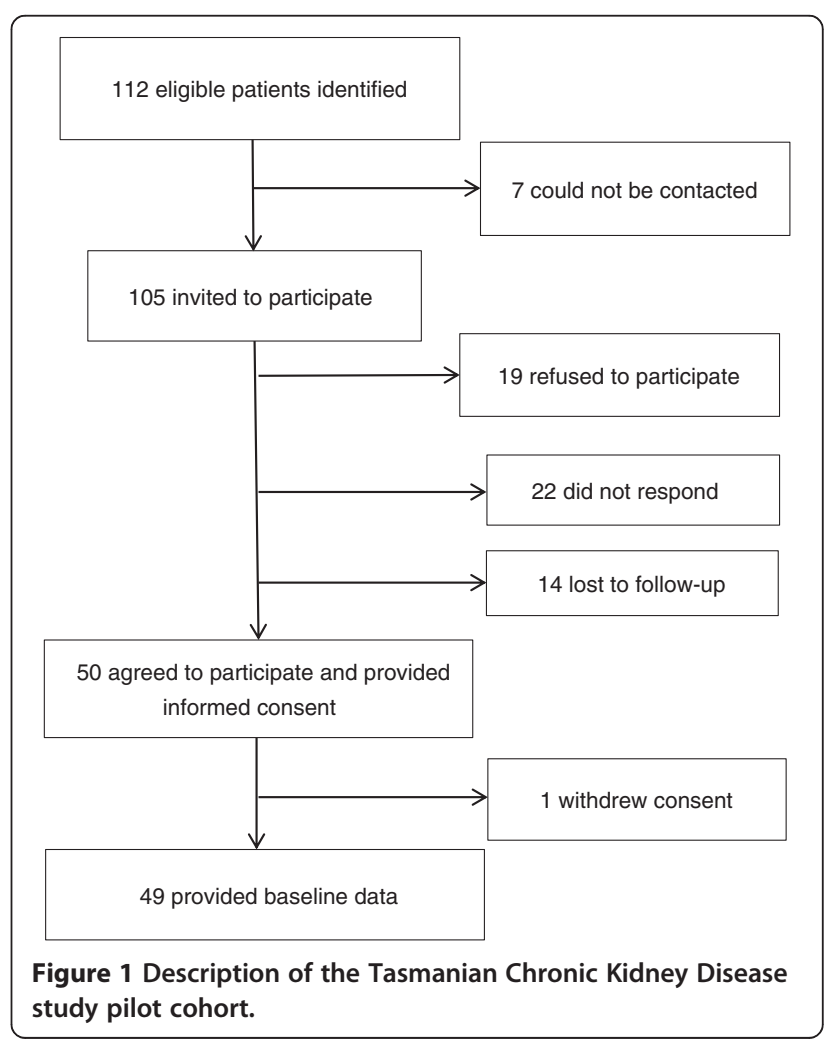


Table 1 Baseline characteristics of pilot participants, the Tasmanian Chronic Kidney Disease study (frequency (\%) unless otherwise indicated)

\begin{tabular}{|c|c|}
\hline Variable & \\
\hline \multicolumn{2}{|l|}{ Sociodemographic } \\
\hline Age $(y r ;$ mean \pm SD) [range] & $73 \pm 10[37-86]$ \\
\hline Female gender & $18(37 \%)$ \\
\hline Education > Year 12 & $12(25 \%)$ \\
\hline Married/living as married & $34(69 \%)$ \\
\hline Retired & $35(71 \%)$ \\
\hline Government pension & $35(71 \%)$ \\
\hline \multicolumn{2}{|l|}{ Index of Disadvantage } \\
\hline Quartile 1 & $13(26 \%)$ \\
\hline Quartile 2 & $13(26 \%)$ \\
\hline Quartile 3 & $10(20 \%)$ \\
\hline Quartile 4 & $14(28 \%)$ \\
\hline \multicolumn{2}{|l|}{ Household income (AUS\$ annual gross) } \\
\hline $1-10,399$ & $1(2 \%)$ \\
\hline $10,400-15,599$ & $6(12 \%)$ \\
\hline $15,600-20,799$ & $10(20 \%)$ \\
\hline $20,800-31,199$ & $17(35 \%)$ \\
\hline $31,200-41,599$ & $2(4 \%)$ \\
\hline $41,600-51,999$ & $1(2 \%)$ \\
\hline $52,000-64,999$ & $2(4 \%)$ \\
\hline $65,000-103,999$ & $0(0 \%)$ \\
\hline$\geq 104,000$ & $2(4 \%)$ \\
\hline Declined to answer & $8(16 \%)$ \\
\hline \multicolumn{2}{|l|}{ Clinical } \\
\hline Depression (PHQ-9 score $\geq 10$ ) & $5(10 \%)$ \\
\hline Anxiety (BAl score $\geq 16$ ) & $4(9 \%)$ \\
\hline Current/former smoker & $27(55 \%)$ \\
\hline$\geq 3$ medical comorbidities & $29(59 \%)$ \\
\hline No. of comorbidities (mean \pm SD) [range] & $3.3 \pm 1.9[1.0-8.0]$ \\
\hline Hypertension & $41(84 \%)$ \\
\hline Systolic BP (mm Hg; mean \pm SD) & $133.3 \pm 22$ \\
\hline Diastolic BP, (mm Hg; mean \pm SD) & $67.8 \pm 12.7$ \\
\hline Diabetes mellitus & $19(39 \%)$ \\
\hline Atherosclerotic heart disease & $21(43 \%)$ \\
\hline Congestive heart failure & $17(35 \%)$ \\
\hline Peripheral vascular disease & $14(29 \%)$ \\
\hline Grip strength, right (kg; mean \pm SD) [range] & $25.9 \pm 8.6[9.0-49.0]$ \\
\hline Grip strength, left (kg; mean \pm SD) [range] & $26.9 \pm 8.8[12.5-47.5]$ \\
\hline 5-metre walk (sec; mean \pm SD) [range] & $8.0 \pm 4.4[5.0-28.0]$ \\
\hline $\mathrm{BMI}\left(\mathrm{kg} / \mathrm{m}^{2} ;\right.$ mean $\left.\pm \mathrm{SD}\right)$ [range] & $30.1 \pm 6.8[21.9-53.5]$ \\
\hline $\mathrm{BMl}<25 \mathrm{~kg} / \mathrm{m}^{2}$ & $8(16 \%)$ \\
\hline BMI $25-29.9$ kg/m² & $23(47 \%)$ \\
\hline
\end{tabular}

Table 1 Baseline characteristics of pilot participants, the Tasmanian Chronic Kidney Disease study (frequency (\%) unless otherwise indicated) (Continued)

\begin{tabular}{lc}
\hline $\mathrm{BMI} \geq 30 \mathrm{~kg} / \mathrm{m}^{2}$ & $15(31 \%)$ \\
Missing & $3(6 \%)$ \\
Laboratory & \\
Hemoglobin (g/L; mean $\pm \mathrm{SD}$ ) [range] & $121.5 \pm 15.0[94.0-163.0]$ \\
Serum creatinine ( $\mu \mathrm{mol} / \mathrm{L}$; mean $\pm \mathrm{SD})$ & $241.1 \pm 61.9[144.0-396.0]$ \\
[range] & \\
eGFR (mL/min $/ 1.73 \mathrm{~m}^{2} ;$ mean $\left.\pm \mathrm{SD}\right)$ [range] & $22.2 \pm 4.9[15.0-30.0]$ \\
\hline
\end{tabular}

Table 1. In summary, participants were predominantly male with a mean age at enrolment of $72.6 \pm 10.2$ years (range 37-86 years). The majority had low educational attainment, were married or living as married and were receiving a Government pension. Around $70 \%$ of participants reported a household income of less than AUS\$31,200 per year. Only three participants were current smokers, while around half $(\mathrm{n}=24)$ were former smokers and over $75 \%$ were overweight or obese. Around $60 \%$ of participants had at least three current medical comorbidities and 39\% had at least four comorbidities. The majority of participants were hypertensive and around $40 \%$ had diabetes mellitus.

Mean depression (PHQ-9) score was $4.4 \pm 5.8$ with five participants $(10 \%)$ meeting the recommended threshold (score $\geq 10$ ) for a clinical diagnosis of major depression. Mean anxiety (BAI) score was $7.8 \pm 7.8$ with $9.3 \%$ of participants reporting at least moderate levels of anxiety (score $\geq 16$ ). Mean social support (MSPSS) score was $5.8 \pm 1.1$ (range 2.6-7.0).

Baseline scores for the health-related quality of life measures (KDQOL-SF 1.3 and EQ-5D) are shown in Table 2. Mean values for the kidney disease targetedscales ranged from 33.7 (work status, $\mathrm{SD}=31.3$ ) to 87.4 (social support, $\mathrm{SD}=21.1$ ) on the range of total possible (0-100) scores. Mean values for the SF-36 scales ranged from 42.7 (role limitations-physical, $\mathrm{SD}=41.9$ ) to 79.6 (social function, $\mathrm{SD}=25.9$ ). The highest percentage of problems reported by participants using the EQ-SD was in the dimension pain/discomfort (61\%) followed by mobility (51\%), usual activities (49\%) and anxiety/depression (27\%).

Associations between psychosocial and biomedical variables, and health-related quality of life subscales (KDQOL-SF) are displayed in Table 3. Consistent inverse associations were observed between depression severity (PHQ-9) and anxiety (BAI) with all of the SF-36 subscales and the majority of kidney disease-targeted subscales. Significant positive correlations were also observed between perceived social support (MSPSS) and the cognitive function and emotional well-being subscales. There were no significant associations between eGFR, hemoglobin, creatinine, and health-related quality of life. 
Table 2 Baseline descriptive statistics for health-related quality of life measures (KDQOL-SF 1.3 and EQ-5D), the Tasmanian Chronic Kidney Disease study

\begin{tabular}{|c|c|}
\hline \multicolumn{2}{|l|}{ Variable } \\
\hline Kidney disease-targeted scales & $($ mean $\pm S D)[\text { range }]^{\wedge}$ \\
\hline Burden of kidney disease & $71.9 \pm 27.0[6.3-100.0]$ \\
\hline Quality of social interaction & $80.3 \pm 19.2[26.7-100.0]$ \\
\hline Cognitive function & $84.1 \pm 21.3[0.0-100.0]$ \\
\hline Symptoms/problems & $76.1 \pm 15.7[31.8-100.0]$ \\
\hline Effects of kidney disease & $86.3 \pm 16.4[14.3-100.0]$ \\
\hline Sexual function & $65.9 \pm 37.1[0.0-100.0]$ \\
\hline Sleep & $63.2 \pm 20.0[5.0-95.0]$ \\
\hline Social support & $87.4 \pm 21.1[0.0-100.0]$ \\
\hline Work status & $33.7 \pm 31.3[0.0-100.0]$ \\
\hline Overall health rating & $64.5 \pm 19.6[20.0-100.0]$ \\
\hline \multicolumn{2}{|l|}{ SF-36 scales } \\
\hline Physical Functioning & $52.1 \pm 29.6[0.0-100.0]$ \\
\hline Role limitations-physical & $42.7 \pm 41.9[0.0-100.0]$ \\
\hline Pain & $66.1 \pm 31.2[0.0-100.0]$ \\
\hline General health & $43.8 \pm 21.2[0.0-85.0]$ \\
\hline Emotional well-being & $76.6 \pm 20.3[8.0-100.0]$ \\
\hline Role limitations-emotional & $63.2 \pm 42.0[0.0-100.0]$ \\
\hline Social function & $79.6 \pm 25.9[0.0-100.0]$ \\
\hline Energy/fatigue & $47.7 \pm 24.5[5.0-90.0]$ \\
\hline SF-36 PCS & $37.7 \pm 10.1[17.5-55.1]$ \\
\hline SF-36 MCS & $50.7 \pm 9.4[19.1-64.1]$ \\
\hline EQ-5D & $\%$ reporting problems \\
\hline Mobility & 51.0 \\
\hline Self-care & 12.2 \\
\hline Usual activities & 49.0 \\
\hline Pain/discomfort & 61.2 \\
\hline Anxiety/depression & 26.5 \\
\hline
\end{tabular}

$\wedge$ No participants were receiving dialysis at time of enrolment so 'Staff encouragement' and 'Patient satisfaction' scales were not completed.

In regards to cognitive function, mean 3MS score was $90.0 \pm 9.2$ with scores ranging from 65 to 100 . Seven participants (15\%) scored below the recommended cutpoint for lower global cognitive functioning (score $<80$ ). One participant had a score at the test ceiling and no participants had scores at the test floor. Mean score on the KDQOL-SF cognitive function subscale was $84.1 \pm 21.3$ with a median score of 93.3 (range 0 to 100). Eighteen (37\%) participants had scores at the test ceiling with only one participant scoring at the test floor.

Decision making preferences indicated that the majority of participants $(63 \%, \mathrm{n}=30)$ prefer to delegate decisions to healthcare professionals (API decision-making subscale, mean score $16.6 \pm 4.3$ ) and just under half of participants $(46 \%, n=22)$ prefer information/education to be provided (API information-seeking subscale, $33.5 \pm$ 3.3). Conversely, around half $(49 \%, n=23)$ reported actively seeking information (KHOS information subscale, $10.7 \pm 2.1)$ with $40 \%$ of participants $(n=18)$ preferentially seeking professional help (KHOS behavioral-involvement subscale, $10.6 \pm 1.6$ ).

Mean ADL score was $5.6 \pm 1.0$ and mean IADL score was $7.2 \pm 1.3$, both indicating reasonably high functioning. Mean KPS score was $77.6 \pm 14.2$ with a range of 40 to 90 also indicating reasonable functional status. Eight participants $(17.4 \%)$ were assigned a score $<70$ indicating that these participants require some level of assistance to carry out daily activities.

At 10-month follow-up in April 2012, one participant had died while eight participants $(16 \%)$ had progressed to KRT and nine participants $(18 \%)$ had progressed to stage 5 CKD without KRT. The remainder of participants remained stable with stage 3 or 4 CKD. For participants not receiving KRT at 10-month follow-up mean serum creatinine was $288 \pm 143 \mu \mathrm{mol} / \mathrm{L}$ with mean eGFR $20 \pm 9 \mathrm{mls} / \mathrm{min} / 1.73 \mathrm{~m}^{2}$.

\section{Discussion}

The Tasmanian Chronic Kidney Disease Study is a prospective cohort study of community-dwelling nondialyzed adults with severe CKD. The study will contribute to the emerging international literature investigating associations between psychosocial factors and kidney disease and, to our knowledge, will be the first study to examine the influence of these factors in adults with CKD prior to dialysis or transplantation. The current analysis describes the clinical, sociodemographic, psychosocial and health-related quality of life characteristics of pilot participants at baseline and their clinical progression at 10-month follow-up. Strengths include the use of a community-based cohort and comprehensive assessment of a range of psychosocial and biomedical variables.

Considerable psychological morbidity was observed in pilot participants with 10\% meeting the threshold for a diagnosis of major depression and $9 \%$ reporting at least moderate levels of clinical anxiety. While numbers are small, this is substantially higher than the 12-month prevalence of affective and anxiety disorders $(4.6 \%$ and $5.2 \%$ respectively) observed in Australians aged 65 to 85 years [42]. Consistent with previous research, less severe depression was associated with higher subjective health-related quality of life [43]. Lower levels of anxiety were also associated with higher quality of life suggesting that anxiety symptoms may be associated with quality of life independent to those of depression. Anxiety and depression are distinct clinical entities that have differing causes, pathophysiological processes, and treatments [44]. Distinguishing between their individual and combined effects will be important to 
Table 3 Spearman correlations between the KDQOL-SF subscales and psychosocial and biomedical variables, the Tasmanian Chronic Kidney Disease study

\begin{tabular}{|c|c|c|c|c|c|c|c|c|c|c|c|}
\hline KDQOL-SF & PHQ-9 & BAI & MSPSS & Age & Hemoglobin & Creatinine & eGFR & CMI & $\mathrm{CF}$ & ADL & KPS \\
\hline \multicolumn{12}{|l|}{ Kidney disease-targeted scales } \\
\hline Burden of kidney disease & $-0.33^{\mathrm{a}}$ & $-0.33^{\mathrm{a}}$ & -0.05 & $0.29^{\mathrm{a}}$ & 0.03 & -0.13 & 0.15 & 0.02 & $0.34^{\mathrm{a}}$ & 0.08 & -0.03 \\
\hline Quality of social interaction & $-0.56^{c}$ & $-0.60^{c}$ & $0.45^{\mathrm{b}}$ & 0.23 & 0.05 & 0.17 & -0.12 & -0.14 & $0.58^{c}$ & $0.37^{\mathrm{b}}$ & 0.12 \\
\hline Cognitive function & $-0.51^{c}$ & $-0.55^{\mathrm{c}}$ & $0.47^{b}$ & 0.05 & -0.16 & 0.03 & -0.06 & -0.20 & 1.00 & $0.36^{\mathrm{a}}$ & 0.21 \\
\hline Symptoms/problems & $-0.61^{c}$ & $-0.61^{\mathrm{c}}$ & 0.11 & 0.15 & -0.05 & -0.07 & 0.11 & -0.16 & $0.45^{\mathrm{b}}$ & 0.21 & 0.18 \\
\hline Effects of kidney disease & $-0.50^{c}$ & $-0.41^{b}$ & 0.13 & -0.004 & -0.22 & -0.09 & -0.02 & $-0.31^{\mathrm{a}}$ & $0.43^{c}$ & 0.23 & 0.17 \\
\hline Sexual function & -0.12 & -0.06 & -0.08 & 0.19 & -0.18 & 0.23 & -0.26 & -0.26 & -0.41 & 0.51 & 0.28 \\
\hline Sleep & $-0.67^{c}$ & $-0.61^{\mathrm{c}}$ & 0.27 & 0.28 & 0.00 & -0.02 & 0.09 & $-0.36^{\mathrm{a}}$ & $0.50^{\mathrm{b}}$ & $0.30^{\mathrm{a}}$ & 0.16 \\
\hline Social support & $-0.34^{\mathrm{a}}$ & -0.25 & $0.54^{c}$ & 0.20 & -0.18 & -0.11 & 0.01 & $-0.36^{\mathrm{a}}$ & $0.49^{c}$ & $0.42^{\mathrm{b}}$ & 0.25 \\
\hline Work status & -0.12 & -0.06 & 0.17 & $-0.32^{\mathrm{a}}$ & 0.04 & 0.15 & -0.21 & -0.14 & 0.09 & 0.13 & 0.23 \\
\hline Overall health rating & $-0.54^{c}$ & $-0.44^{\mathrm{b}}$ & 0.24 & 0.03 & -0.22 & 0.01 & -0.14 & $-0.46^{b}$ & $0.36^{\mathrm{a}}$ & $0.29^{\mathrm{a}}$ & $0.30^{\mathrm{a}}$ \\
\hline \multicolumn{12}{|l|}{ SF-36 scales } \\
\hline Physical functioning & $-0.46^{b}$ & $-0.52^{c}$ & 0.09 & -0.16 & 0.15 & 0.05 & -0.02 & $-0.38^{\mathrm{a}}$ & 0.23 & $0.33^{\mathrm{a}}$ & $0.10^{\mathrm{b}}$ \\
\hline Role limitations-physical & $-0.45^{\mathrm{b}}$ & $-0.42^{\mathrm{b}}$ & -0.06 & -0.14 & 0.00 & 0.05 & -0.03 & -0.25 & $0.31^{\mathrm{a}}$ & 0.26 & 0.23 \\
\hline Pain & $-0.37^{b}$ & $-0.60^{c}$ & 0.12 & 0.08 & 0.14 & 0.05 & 0.01 & $-0.30^{\mathrm{a}}$ & $0.29^{\mathrm{a}}$ & 0.20 & 0.29 \\
\hline General health & $-0.72^{c}$ & $-0.63^{c}$ & 0.13 & 0.19 & 0.08 & 0.04 & -0.001 & $-0.48^{\mathrm{c}}$ & $0.30^{\mathrm{a}}$ & 0.16 & 0.27 \\
\hline Emotional well-being & $-0.50^{c}$ & $-0.64^{c}$ & $0.41^{\mathrm{b}}$ & 0.14 & 0.11 & 0.24 & -0.21 & -0.27 & $0.59^{\mathrm{b}}$ & $0.32^{\mathrm{a}}$ & 0.15 \\
\hline Role limitations - emotional & $-0.35^{\mathrm{a}}$ & $-0.37^{\mathrm{a}}$ & -0.11 & 0.11 & 0.24 & -0.06 & 0.20 & 0.14 & 0.06 & -0.03 & 0.09 \\
\hline Social function & $-0.67^{c}$ & $-0.62^{c}$ & 0.18 & 0.06 & 0.17 & 0.09 & -0.10 & -0.25 & $0.57^{b}$ & 0.28 & 0.25 \\
\hline Energy/fatigue & $-0.65^{c}$ & $-0.68^{c}$ & 0.22 & -0.06 & -0.05 & 0.08 & -0.07 & $-0.33^{\mathrm{a}}$ & $0.40^{\mathrm{b}}$ & 0.15 & $0.34^{\mathrm{a}}$ \\
\hline SF36 physical composite score & $-0.48^{b}$ & $-0.61^{\mathrm{c}}$ & 0.15 & 0.08 & -0.04 & 0.15 & -0.12 & -0.29 & 0.26 & 0.18 & 0.14 \\
\hline SF36 mental composite score & $-0.61^{c}$ & $-0.58^{c}$ & 0.26 & 0.23 & 0.05 & 0.22 & -0.11 & -0.12 & $0.47^{\mathrm{b}}$ & 0.30 & 0.21 \\
\hline
\end{tabular}

${ }^{a} p<0.05 ;{ }^{b} p<0.01 ;{ }^{c} p<0.001$.

ADL, Activities of Daily Living; BAI, Beck Anxiety Inventory; CF, cognitive function subscale; CMI, Charlson comorbidity index; eGFR, estimated Glomerular Filtration Rate; KPS, Karnofsky Performance Scale index; MSPSS, Multidimensional Scale of Perceived Social Support; PHQ-9, Patient Health Questionnaire.

understanding the mechanisms by which psychological states are associated with kidney function. While perceived social support (MSPSS) was comparable to population norms [45], participants rated the quality of their social interactions (KDQOL-SF) similarly to patients on dialysis [29].

Substantial loss of physical well-being was also observed in pilot participants with self-reported physical functioning, general health and energy/fatigue (KDQOL$\mathrm{SF}$ ) being comparable to patients on dialysis [29]. Similarly, all SF-36 subscale scores, except for the emotional well-being and the mental component summary scores, were lower than the age and sex standardized mean scores in the Australian population [46]. Further, over $60 \%$ of participants reported experiencing pain/discomfort and around 50\% endorsed problems with mobility and usual activities, further highlighting the considerable physical symptom burden experienced by current participants. As previously observed, there were no significant associations between renal function parameters and health-related quality of life [47]. A possible explanation is that while the disease burden in current participants is substantial they may deny the impact of their illness as a coping mechanism.

There are several limitations of the current investigation that should be considered. As this study was designed as a pilot to assess the feasibility of a larger cohort study a formal power calculation was not performed. Results should therefore be treated as preliminary and interpreted with caution. In addition, due to the cross-sectional design, the direction of causality cannot be inferred. Further, depressive and anxiety symptoms were ascertained via self-report rather than standardized clinical interviews which may underestimate the prevalence of these disorders $[18,48]$. However all self-report measures used in the current study have been validated in renal populations and are brief and easy to administer and interpret. Finally, although non-participants did not differ from participants in terms of age, gender and socio-economic status, the potential for non-response bias remains.

While the participation rate in the current study appears low, it is comparable to previous pilot studies involving adults with chronic disease [49] and consistent with evidence that those with poorer health status, lower 
levels of functioning and higher levels of psychological distress are less likely to participate in population-based research [50]. Our aim is to recruit 700 participants for the state-wide prospective cohort study. This represents $30 \%$ of the target population [16] which is well below the current response rate of $47 \%$. Despite this, efforts to increase participation and limit attrition will be important in ensuring adequate statistical power to examine associations between the key variables of interest. Future strategies involve recruiting face-to-face via treating physicians and assessing participants during usual care thereby increasing response rates and reducing participant burden.

Australia will spend over AUS $\$ 11$ billion on dialysis and kidney transplantation in the next 10 years [51]. According to Australian registry data, for every new case that receives dialysis another patient with ESKD dies without having received it [52]. Further, for those who do commence dialysis, around $37 \%$ will die via withdrawal from treatment [53]. For these patients, medical management may involve a shift from efforts to prolong life to a more supportive/palliative care model focusing on care, quality of life and symptom control. As a patient proceeds from the community to hospital treatment, possible dialysis, then death, there are critical opportunities to improve health and quality of life by identifying and then modifying psychological and social health determinants.

\section{Conclusions}

This paper describes the study design, baseline characteristics and initial follow-up of pilot participants in the Tasmanian Chronic Kidney Disease study. Current results indicate that ongoing recruitment and prospective assessment of this cohort is feasible and has the potential to evaluate the relative impact of both biomedical and psychosocial factors on rate of disease progression, use of KRT, and salient personal and economic outcomes. Findings from this study have the potential to provide an evidence base for revising healthcare decision making and treatment pathways in order to optimize the care of patients with CKD.

\section{Competing interests}

The authors declare that they have no competing interests.

\section{Authors' contributions \\ CM drafted the manuscript, performed the statistical analysis and incorporated critical revisions of the manuscript. AV, CB, MN, AP, MA and JS all contributed to the study design, interpretation of the data and critical revisions of the manuscript. MJ contributed to the study design, acquisition and interpretation of the data, and drafting of the manuscript. All authors read and approved the final manuscript.}

\section{Acknowledgements}

This study was funded by a Jacquot Research Establishment Award from the Royal Australasian College of Physicians. AV and LB are supported by National Health and Medical Research Council fellowships. The authors gratefully acknowledge the contribution of research staff, staff of the Renal Unit of the Royal Hobart Hospital, participating renal physicians and general practitioners, and all study participants.

\section{Author details}

${ }^{1}$ Menzies Research Institute Tasmania, University of Tasmania, Hobart, Tasmania, Australia. ${ }^{2}$ Royal Hobart Hospital, Hobart, Tasmania, Australia. ${ }^{3}$ School of Psychology, Faculty of Health Sciences, University of Tasmania, Hobart, Tasmania, Australia. ${ }^{4}$ School of Medicine, University of Tasmania, Hobart, Tasmania, Australia.

Received: 12 October 2012 Accepted: 9 April 2013

Published: 12 April 2013

\section{References}

1. Katon W, Lin E, Korff M, Ciechanowski P, Ludman E, Young B, Peterson D, Rutter C, McGregor M, McCulloch D: Collaborative care for patients with depression and chronic illnesses. New Engl J Med 2010, 363:2611-2620.

2. Sareen J, Jacobi F, Cox B, Belik S, Clara I, Stein M: Disability and poor quality of life associated with comorbid anxiety disorders and physical conditions. Arch Intern Med 2006, 166:2109-2116.

3. House J, Landis K, Umberson D: Social relationships and health. Science 1988, 241:540-545.

4. Fekete E, Antoni M, Schneiderman N: Psychosocial and behavioral interventions for chronic medical conditions. Curr Opin Psychiatry 2007, 20:152-157.

5. Australian Institute of Health and Welfare (AlHW): Chronic Kidney Disease in Australia. Canberra: AlHW; 2005.

6. Lopes A, Bragg J, Young E, Goodkin D, Mapes D, Combe C, Piera L, Held P, Gillespie B, Port F: Depression as a predictor of mortality and hospitalization among hemodialysis patients in the United States and Europe. Kidney Int 2002, 62:199-207.

7. Hedayati SS, Bosworth HB, Briley LP, Sloane RJ, Pieper CF, Kimmel PL, Szczech LA: Death or hospitalization of patients on chronic hemodialysis is associated with a physician-based diagnosis of depression. Kidney Int 2008, 74:930-936.

8. Kimmel PL, Peterson RA, Weihs KL, Simmens SJ, Alleyne S, Cruz I, Veis JH: Multiple measurements of depression predict mortality in a longitudinal study of chronic hemodialysis outpatients. Kidney Int 2000, 57:2093-2098.

9. Hedayati S, Minhajuddin A, Afshar M, Toto R, Trivedi M, Rush A: Association between major depressive episodes in patients with chronic kidney disease and initiation of dialysis, hospitalization, or death. JAMA 2010, 303:1946-1953.

10. Tsai Y-C, Chiu Y-W, Hung C-C, Hwang S-J, Tsai J-C, Wang S-L, Lin M-Y, Chen $\mathrm{H}-\mathrm{C}$ : Association of symptoms of depression with progression of CKD. Am J Kidney Dis 2012, 60:54-61.

11. Kop WJ, Seliger SL, Fink JC, Katz R, Odden MC, Fried LF, Rifkin DE, Sarnak MJ, Gottdiener JS: Longitudinal association of depressive symptoms with rapid kidney function decline and adverse clinical renal disease outcomes. CJASN 2011, 6:834-844.

12. Cukor D, Coplan J, Brown C, Friedman S, Newville H, Safier M, Spielman LA, Peterson RA, Kimmel PL: Anxiety disorders in adults treated by hemodialysis: a single-center study. Am J Kidney Dis 2008, 52:128-136.

13. Kimmel PL, Peterson RA, Weihs KL, Simmens SJ, Alleyne S, Cruz I, Veis JH: Psychosocial factors, behavioral compliance and survival in urban hemodialysis patients. Kidney Int 1998, 54:245-254.

14. Untas A, Thumma J, Rascle N, Rayner H, Mapes D, Lopes AA, Fukuhara S, Akizawa T, Morgenstern H, Robinson BM, Pisoni RL, Combe C: The associations of social support and other psychosocial factors with mortality and quality of life in the Dialysis Outcomes and Practice Patterns Study. CJASN 2011, 6:142-152.

15. Thong MS, Kaptein AA, Krediet RT, Boeschoten EW, Dekker FW: Social support predicts survival in dialysis patients. Nephrol Dial Transplant 2007, 22:845-850

16. Jose MD, Otahal P, Kirkland G, Blizzard L: Chronic kidney disease in Tasmania. Nephrology 2009, 14:743-749.

17. Clinical Audit Tool (CAT): [http://www.clinicalaudit.com.au/].

18. Kroenke K, Spitzer R, Williams J: The PHQ-9: validity of a brief depression severity measure. J Gen Intern Med 2001, 16:606-613.

19. Beck A, Epstein N, Brown G, Steer R: An inventory for measuring clinical anxiety: psychometric properties. J Consult Clin Psychol 1988, 56:893-897. 
20. Zimet GD, Dahlem NW, Zimet SG, Farley GK: The Multidimensional Scale of Perceived Social Support. J Pers Assess 1988, 52:30-41.

21. Watnick S, Wang P, Demadura T, Ganzini L: Validation of 2 depression screening tools in dialysis patients. Am J Kidney Dis 2005, 46:919-924.

22. Beck A, Steer R: Beck Anxiety Inventory manual. 2nd edition. San Antonio, TX: Psychological Corporation; 1993.

23. Wetherell J, Areán P: Psychometric evaluation of the Beck Anxiety Inventory with older medical patients. Psychol Assess 1997, 9:136-144.

24. Spinale J, Cohen S, Khetpal P, Peterson R, Clougherty B, Puchalski C, Patel S, Kimmel P: Spirituality, social support, and survival in hemodialysis patients. CJASN 2008, 3:1620-1627.

25. Australian Bureau of Statistics (ABS): Census of population and housing: socioeconomic indexes for areas (SEIFA). Canberra: ABS; 2001.

26. Liu J, Huang Z, Gilbertson DT, Foley RN, Collins AJ: An improved comorbidity index for outcome analyses among dialysis patients. Kidney Int 2009, 77:141-151.

27. National Heart Lung and Blood Institute: Clinical Guidelines for the Identification, Evaluation and Treatment of Overweight and Obesity in Adults: The Evidence Report. Bethesda, MD: National Heart, Lung, and Blood Institute; 1998

28. Teng E, Chui $\mathrm{H}$ : The Modified Mini-Mental State (MMS) examination. J Clin Psychiatry 1987, 48:314-318.

29. Hays R, Kallich J, Mapes D, Coons S, Carter W: Development of the Kidney Disease Quality of Life (KDQOL) Instrument. Qual Life Res 1994, 3:329-338.

30. Karnofsky D, Burchenal J: The clinical evaluation of chemotherapeutic agents in cancer. In Evaluation of Chemotherapeutic Agents in Cancer. Edited by McLeod C. New York: Columbia University Press; 1949:191-205.

31. Katz S, Ford A, Moskowitz R, Jackson B, Jaffe M: Studies of illness in the aged. The index of ADL: a standardized measure of biological and psychosocial function. JAMA 1963, 185:914-919.

32. Lawton M, Brody E: Assessment of older people: self-maintaining and instrumental activities of daily living. Gerontologist 1969, 9:179-186.

33. Ende J, Kazis L, Ash A, Moskowitz M: Measuring patients' desire for autonomy: decision making and information-seeking preferences among medical patients. J Gen Intern Med 1989, 4:23-30.

34. Krantz D, Baum A, Wideman M: Assessment of preferences for selftreatment and information in health care. J Pers Soc Psychol 1980, 39:977-990.

35. Kurella M, Luan J, Yaffe K, Chertow GM: Validation of the Kidney Disease Quality of Life (KDQOL) Cognitive Function subscale. Kidney Int 2004, 66:2361-2367.

36. McClellan WM, Anson C, Birkeli K, Tuttle E: Functional status and quality of life: predictors of early mortality among patients entering treatment for end stage renal disease. J Clin Epidemiol 1991, 44:83-89.

37. Levey $\mathrm{A}: \mathrm{K} / \mathrm{DOQ}$ clinical practice guidelines for chronic kidney disease: evaluation, classification, and stratification. Am J Kidney Dis 2002, 39:266.

38. Levey A, Coresh J, Greene T, Stevens L, Zhang Y, Hendriksen S, Kusek J, Van Lente $F$ : Using standardized serum creatinine values in the modification of diet in renal disease study equation for estimating glomerular filtration rate. Ann Intern Med 2006, 145:247-254.

39. Cleemput I, Kesteloot K, Moons P, Vanrenterghem Y, Van Hooff JP, Squifflet J-P, De Geest S: The construct and concurrent validity of the EQ-5D in a renal transplant population. Value in Health 2004, 7:499-509.

40. Liem Y, Bosch J, Myriam Hunink M: Preference-based quality of life of patients on renal replacement therapy: a systematic review and metaanalysis. Value in Health 2008, 11:733-741.

41. Wyld M, Morton RL, Hayen A, Howard K, Webster AC: A systematic review and meta-analysis of utility-based quality of life in chronic kidney disease treatments. PLoS Med 2012, 9:e1001307.

42. Australian Bureau of Statistics: National Survey of Mental Health and Wellbeing: summary of results 2007. Canberra: ABS; 2008.

43. Porter A, Fischer M, Brooks D, Bruce M, Charleston J, Cleveland W, Dowie D, Faulkner M, Gassman J, Greene T, Hiremath L, Kendrick C, Kusek J, ThornleyBrown D, Wang X, Norris K, Unruh M, Lash J: Quality of life and psychosocial factors in African Americans with hypertensive chronic kidney disease. Translational Research 2012, 159:4-11.

44. Nutt D, Stein D: Understanding the neurobiology of comorbidity in anxiety disorders. CNS Spectrums 2006, 11:13-20.

45. Kazarian SS, McCabe SB: Dimensions of social support in the MSPSS: factorial structure, reliability, and theoretical implications. J Community Psychol 1991, 19:150-160.
46. Australian Bureau of Statistics: National Health Survey: SF36 population norms, Australia. Canberra: ABS; 1995.

47. Cohen S, Patel S, Khetpal P, Peterson R, Kimmel P: Pain, sleep disturbance, and quality of life in patients with chronic kidney disease. CJASN 2007, 2:919-925

48. Leyfer $\mathrm{O}$, Ruberg J, Woodruff-Borden J: Examination of the utility of the Beck Anxiety Inventory and its factors as a screener for anxiety disorders. J Anxiety Disord 2006, 20:444-458

49. Schröder FH, Denis LJ, Kirkels W, De Koning HJ, Standaert B: European randomized study of screening for prostate cancer. Progress report of Antwerp and Rotterdam Pilot studies. Cancer 1995, 76:129-134.

50. Galea S, Tracy M: Participation rates in epidemiologic studies. Ann Epidemiol 2007, 17:643-653.

51. Cass A, Chadban S, Craig J, Howard H, McDonald S, Salkeld G, White S: The economic impact of end-stage kidney disease in Australia: Projections to 2020 Melbourne: Kidney Health Australia; 2010.

52. Australian Institute of Health and Welfare (AlHW): End-stage kidney disease in Australia: total incidence, 2003-2007. Canberra: AlHW; 2011

53. Chan HW, Clayton PA, McDonald SP, Agar JW, Jose MD: Risk factors for dialysis withdrawal: an analysis of the Australia and New Zealand Dialysis and Transplant (ANZDATA) Registry, 1999-2008. CJASN 2012, 7:775-781.

doi:10.1186/1471-2369-14-83

Cite this article as: McKercher et al:: Psychosocial factors in adults with chronic kidney disease: characteristics of pilot participants in the Tasmanian Chronic Kidney Disease study. BMC Nephrology 2013 14:83.

\section{Submit your next manuscript to BioMed Central and take full advantage of:}

- Convenient online submission

- Thorough peer review

- No space constraints or color figure charges

- Immediate publication on acceptance

- Inclusion in PubMed, CAS, Scopus and Google Scholar

- Research which is freely available for redistribution

Submit your manuscript at www.biomedcentral.com/submit
C Biomed Central 